\title{
LA PEROXIDACIÓN DE LÍPIDOS EN BRIÓFITOS ACUÁTICOS SOMETIDOS A CONTAMINACIÓN ORGÁNICA
}

\author{
J. Martínez Abaigar ', E. Núñez Olivera' y M. Sánchez-Díaz ${ }^{2}$ \\ 1. Area de Biología Vegetal, Universidad de La Rioja, Luis de Ulloa 20, 26004 Logroño, España. \\ ${ }^{2}$. Dpto. Fisiología Vegetal, Universidad de Navarra, Apdo. 273, 31080 Pamplona, España.
}

Palabras clave: Macrófitos, peroxidacióri de Iípidos, contaminación de aguas, bioindicadores.

\section{RESUMEN}

Se utiliza la peroxidación de Iípidos como indicador de contaminación orgánica en el río Iregua (La Rioja, España), sobre Fontinalis antipyretica Hedw., un briófito acuático trasplantado desde un lugar libre de contaminación en el curso alto del río a dos estaciones de muestreo, una contaminada y otra no contaminada. La técnica consiste en la valoración espectrofotométrica del malondialdehido (MDA), un producto de la peroxidación de Iípidos, en el tejido vegetal.

El contenido de MDA mostró buena correlación con la $\mathrm{DBO}_{5}$ a lo largo del tiempo en la estación contaminada, por lo que aparece como un buen indicador de la senescencia vegetal causada por la contaminación orgánica.

\section{ABSTRACT \\ LIPID PEROXIDATION IN AQUATIC BKYOPHYTES AFFECTED BY ORGANIC POLLUTION}

Lipid peroxidation in the aquatic bryophyte Fontinalis antipyretica Hedw., estimated through the spectrophotometric determination of malondialdehyde (MDA), has been used as an organic pollution indicator in the Iregua river (La Rioja, Spain). The bryophyte was transplanted from a high-course non-polluted place to another non-polluted station located in the middle course (Villanueva de Carneros, control station) and to a lower-course polluted one (Alberite).

During the organic pollution episodes of Alberite, MDA level clearly increased and it also showed a good correlation with BOD, all over the experiment. The utilization of lipid peroxidation as indicator of plant senescence caused by organic pollution is discussed.

\section{INTRODUCCIÓN}

Tradicionalmente se ha resaltado el papel del oxígeno en la respiración vegetal. Sin embargo, sólo en los últimos años se les ha prestado atención a otros procesos relacionados directamente con el oxígeno y de trascendental importancia para plantas en estado de tensión. Bajo ciertas condiciones el oxígeno actúa como tóxico y los vegetales deben desplegar mecanismos defensivos contra él. Las principales especies activas de oxígeno que resultan tóxicas son el anión superóxido, el radical hidroxilo, el peróxido de hidrógeno y el oxígeno singlete (LESHEM, 1981). Su aparición puede estar causada por fenómenos de tres tipos (ELSTNER, 1982): antropogénicos (contaminación por herbicidas, metales pesados, SO, CO, ozono, "smog"), parasíticos (infecciones) y ambientales (solarización, estreses térmicos, sequía, salinidad). Su toxicidad radica en las reacciones de foto-oxidación que son capaces de producir con moléculas-diana: proteínas, ácidos nucleicos, Iípidos, pigmentos, y especialmente carbohidratos y ácidos grasos poliinsaturados de membranas (LESHEM, 1981). Por ello están relacionados íntimamente con la senescencia vegetal. Esta influencia sc cuantifica mediante diversas técnicas, entre ellas la peroxidación dc los Iípidos de membrana y la actividad de los enzimas que intervienen en el metabolismo de dichas especies activas (DHINDSA \& MATOWE, 1981; DHINDSA et ul., 1981 y 1982). Pero pocas veces se han utilizado estas técnicas en monitorización de contaminación (SHIMAZAKI et al., 1980; CHIA et al., 1984). 
Los briofitos, y en general las criptógamas, son reconocidos como grupos biológicos de gran valor bioindicador (WIELGOLASKI, 1975; ANDO \& MATSUO, 1984; MÜHLE, 1984). Sus ventajas con respecto a las fanerógamas en aspectos de contaminación residen en numerosos factores: amplia distribución, tanto en los diversos hábitats como en el tiempo (pueden muestrearse durante todo el año); gran sensibilidad a los contaminantes, con umbrales de ataque inferiores a las fanerógamas, por la carencia de barreras estructurales que impidan la absorción de los contaminantes; longevidad, que permite estudios integrados en el tiempo sin interrupciones estacionales; crecimiento lento y capacidad de acumulación selectiva de ciertos elementos, como metales pesados; simplicidad estructural, etc.

Los briofitos son capaces de detectar numerosos tipos de contaminación, tanto en ambientes terrestres como acuáticos. Entre los ensayos experimentales efectuados sobre briofitos como indicadores de contaminación atmosférica destacan los dedicados al SO, metales pesados, lluvia ácida, hidrocarburos, productos radiactivos, herbicidas, fluoruros, etc. (revisiones en LEBLANC \& RAO, 1974 y 1975; RAO, 1982; BROWN, 1984; MÜHLE, 1984). En el medio acuático, destacaremos las aportaciones de EMPAIN (1973 y 1978) referidas a parámetros físico-químicos del agua, los trabajos de WEHR et al. (1983) en metodología sobre metales pesados, KIRCHMANN \& LAMBINON (1973) y MAUREL-KERMARREC et al. (1983) en contaminación radiactiva, y PEÑUELAS (1984) en contaminación orgánica. Las técnicas utilizadas han sido variadas: estudios florísticos y sinecológicos (LEBLANC \& DE SLOOVER, 1970; WINNER \& BEWLEY, 1978 a y b); análisis de contaminantes en tejidos (entre otros, CASE \& KROUSE, 1980; SUMMERLING, 1984; BROWN et al., 1986); extracción de pigmentos (PEÑUELAS, 1984); trasplantes (LEBLANC \& RAO, 1973; BENSON-EVANS \& WILLIAMS, 1976; PEÑUELAS, 1984); cultivos artificiales (WINNER \& BEWLEY, 1983; GOOSENS, 1976 a y b, 1979), etc. La peroxidación de Iípidos, sin embargo, sólo ha sido estudiada en relación con procesos de sequía (DHINDSA \& MATOWE, 1981).

No hay que olvidar, por otra parte, la relación que se establece generalmente entre la contaminación atmosférica y la de las aguas (WIELGOLASKI, 1975; MARGALEF, 1983; ROELOFS, 1986). Diversos contaminantes atmosféricos acaban por depositarse en sistemas acuáticos, bien sea por acción de la lluvia, el viento, la fusión de nieve contaminada, la escorrentía... Es conocida la potenciación de la acidificación en sistemas límnicos por la lluvia ácida, mucho más grave en lagos con escasa reserva alcalina (MARGALEF, 1983). Además, la cuenca fluvial, aunque comprende distintos ecosistemas, forma una uni- dad ecológica (MARGALEF, 1974), y es importante atender a la influencia de la contaminación atmosférica sobre la calidad de las aguas próximas y sobre su vegetación macrofítica.

El musgo utilizado, Fontinalis antipyretica Hedw., se trasplantó desde el curso alto del río Iregua (La Rioja) a dos estaciones aguas abajo, una de aguas limpias y otra con problemas episódicos de contaminación orgánica. La peroxidación de lípidos en los tejidos briofíticos se tomó como indicador del deterioro sufrido por el musgo como consecuencia de los pulsos de contaminación.

\section{MATERIAL Y MÉTODOS}

\section{Recolección y trasplante}

El musgo objeto de la experiencia (Fontinalis antipyreticu Hedw.) fue recolectado en mayo de 1986 en el arroyo de los Senestillos, cerca de Pajares (La Rioja), a $1350 \mathrm{~m}$ de altitud. El material recogido fue rápidamente trasplantado a una estación libre de contaminación (Villanueva de Cameros, $900 \mathrm{~m}$ de altitud) y a una contaminada (Alberite, $440 \mathrm{~m}$ ), y fue introducido en bolsas de malla de plástico verde, suficientemente laxa para evitar un déficit excesivo de radiación luminosa. Las bolsas se fijaban mediante hilo de pita a un mástil metálico, y éste era clavado en el lecho del río de manera que las bolsas, arrastradas por la corriente, se mantuviesen a una profundidad aproximada de $20 \mathrm{~cm}$. El procedimiento es el de BENSON-EVANS \& WILLIAMS (1976) simplificado. Se realizó una recolección semanal en el periodo 12 de mayo al 13 de julio de 1986. Los lugares seleccionados para el trasplante en Villanueva y Alberite se eligieron teniendo en cuenta criterios de similaridad de condiciones ambientales (luminosidad, velocidad de corriente), accesibilidad y prevención de destrucción deliberada del montaje.

\section{Peroxidación de lípidos}

El material trasplantado era transportado al laboratorio en nevera portátil a $5^{\circ} \mathrm{C}$ de temperatura, y era lavado en agua destilada y almacenado en oscuridad a $5^{\circ} \mathrm{C}$. Dentro de las 48 horas siguientes a la recolección se seleccionaban los $4 \mathrm{~cm}$ apicales de los tallitos de Fontinalis. Se obtenía un extracto en mortero con aproximadamente $250 \mathrm{mg}$ de peso fresco y ácido tricloroacético (TCA) al $0,1 \%$, filtrando a continuación por cuatro capas de gasa. A $2 \mathrm{ml}$ del filtrado se le añadían $2 \mathrm{ml}$ de ácido tiobarbitúrico (TBA) al $0,1 \%$ en TCA al $20 \%$, y se calentaba la mezcla a $95^{\circ} \mathrm{C}$ durante 30 minutos. Se enfriaba rápidamente en bano de hielo y se filtraba por filtros Whatman GF/C. La con- 
centración de malondialdehido (MDA), un producto resultante de la peroxidación de Iípidos, se calculaba por diferencia entre las absorbancias a $532 \mathrm{~nm}$ y $600 \mathrm{~nm}$ (inespecífica), teniendo en cuenta que el coeficiente de extinción del MDA es de $155 \mathrm{mM}^{1}$ cm.' (DHINDSA \& MATOWE, 1981; DHINDSA et al., 1981 y 1982). Las determinaciones se hacían por duplicado. Paralelamente al pesado del tejido fresco, dos muestras se introducían en estufa a $80^{\circ} \mathrm{C}$ durante 24 horas para calcular el peso seco y el factor de conversión. Los resultados se expresan en función del peso seco.

En una serie previa de pruebas de la técnica con otro briófito, Dicranum scoparium Hedw., se observó que en la reacción MDA-TBA en caliente se detectaban dos máximos de absorbancia, uno a $532 \mathrm{~nm}$ y otro a $445 \mathrm{~nm}$ (Fig. 1). El primero se debía al MDA, y el segundo estaba originado por reacciones colorimétricas no específicas del TBA en caliente con gliceraldehido, azúcares, ácidos orgánicos y otros compuestos (SAWICKI et al., 1963). Este máximo a $445 \mathrm{~nm}$ no interfería en ningún caso con el máximo a $532 \mathrm{~nm}$.

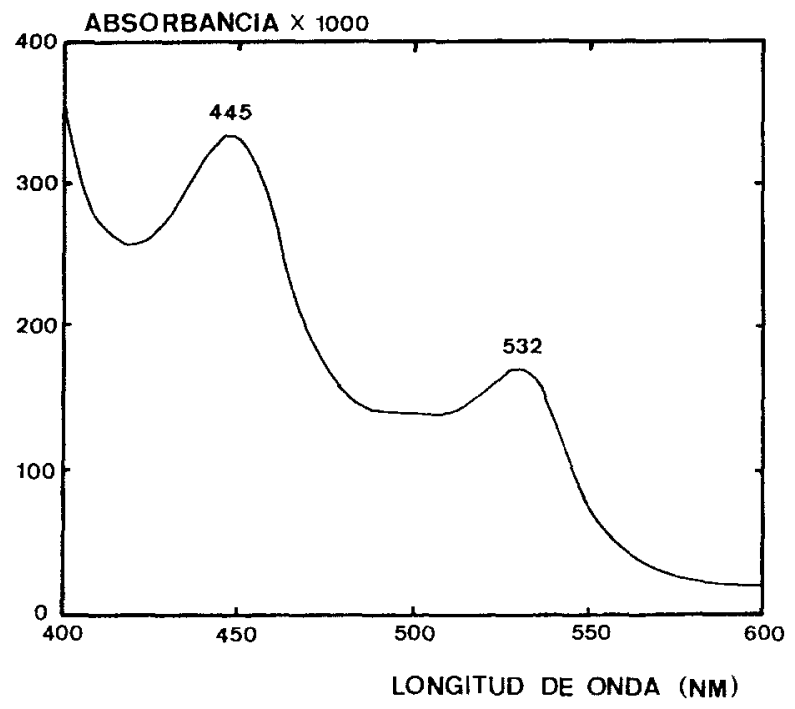

FIGURA 1.- Espectro de absorción derivado de la reacción en caliente del ácido tiobarbitúrico (TBA) con el extracto de Dicranum scoparium.

FIGURE 1.- Absorption spectrum derived from the hot reaction of tiobarbituric acid (TBA) with the extract of Dicranum scoparium.

\section{Análisis de aguas}

Las muestras de agua se tomaban semanalmente, de forma paralela a las recolecciones de material vegetal en Villanueva y Alberite. Los análisis realizados incluían un total de 17 variables habituales (MARTINEZ ABAIGAR, 1989). La $\mathrm{DBO}_{5}$ se calculó midiendo el contenido de oxígeno (electrodo Orion) antes y después de incubar la muestra de agua a $20^{\circ} \mathrm{C}$ durante cinco días a la oscuridad.

\section{Análisis estadístico}

A la concentración de MDA se le aplicó una t de Student entre las dos estaciones (Alberite y Villanueva) en las diferentes semanas de la experiencia.

\section{RESULTADOS}

Durante el periodo muestreado, Villanueva estaba libre de contaminación orgánica (DBO, entre 0,5 y $2,5 \mathrm{mg} \mathrm{l}^{-1}$ ), mientras que Alberite sufrió dos pulsos de contaminación (Fig. 2).
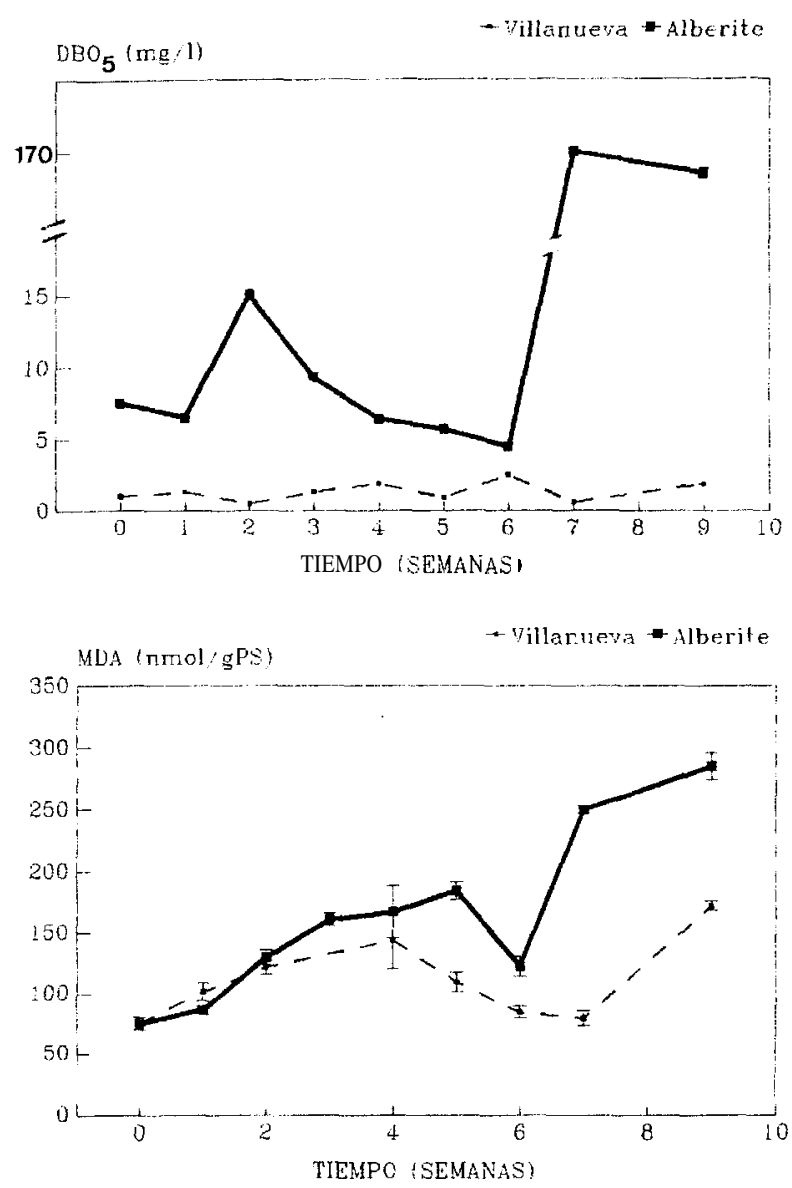

FIGURA. 2.- Variación en la DBO, del agua y en el contenido de malondialdehido (MDA, media \pm error estándar) de Fontinalis antipyretica durante el periodo muestreado, en la estación contaminada (Alberite) y en la no contaminada (Villanueva).

FIGURE. 2.- Changes in $\mathrm{BOD}_{5}$ of the water and malondialdebyde content (MDA, mean \pm standard error) of Fontinalis antipyretica in the sampled period, in the polluted station (Alberite) and in the non-polluted one (Villanueva). 
La DBO, mostró en Alberite un máximo relativo en la segunda semana, con 15,1 mg l', y un máximo absoluto en las semanas séptima y novena con valores en tomo a $170 \mathrm{mg} 1^{1}$.

Los valores de MDA obtenidos quedan reflejados en la Fig. 2. El material de partida, recolectado en Pajares y no trasplantado, tenía una concentración de MDA de 76 nmol g ${ }^{2}$ PS. En las primeras semanas el MDA aumentó en el briófito en ambas estaciones de trasplante, sin diferencias significativas entre ellas. En Villanueva descendió posteriormente hasta volver a alcanzar los valores originales en la séptima semana, con 80 nmol g PS, y se incrementó en la semana 9 hasta 172 . En Alberite se registró un significativo mínimo relativo de MDA en la sexta semana, coincidiendo con el mínimo absoluto de $\mathrm{DBO}_{5}\left(4,5 \mathrm{mg} \mathrm{l}^{-1}\right)$, y bruscamente ascendió hasta valores de 250 y $285 \mathrm{nmol}^{-1} \mathrm{PS}$ en la séptima y novena semanas, paralelamente al aumento de la DBO,. Las concentraciones de MDA en Villanueva y Alberite eran significativamente diferentes $(\mathrm{p}<0,001)$ a partir de la quinta semana de trasplante. La correlación entre la DBO, y la concentración de MDA resultó muy alta en Alberite $(r=0,85, p<0,01)$, pero no en Villanueva $(r=$ 0,19 , no significativa).

\section{DISCUSIÓN}

Se han descrito en la bibliografía numerosos procesos que provocan la aparición de especies activas de oxígeno en los vegetales, tanto en cloroplastos aislados (HEATH \& PACKER, 1965 y 1968) como en órganos (LESHEM, 1981; DHINDSA \& MATOWE, 1981; DHINDSA et al., 1981 y 1982; ELSTNER, 1982). Entre ellos figuran diversos tipos de contaminación (SHIMAZAKI et al., 1980; CHIA et al., 1984), pero no la orgánica. Tampoco se había ensayado nunca la técnica sobre macrófitos.

En las primeras semanas del experimento, la evolución del MDA es parecida en Alberite y Villanueva, pues Fontinalis antipyretica acusó sólo ligeramente la contaminación de esas semanas, y el aumento de peroxidación de lípidos se debería al estrés provocado por el trasplante. Posteriormente, la severa contaminación de las semanas finales, con periodos de anoxia intensos y prolongados, provocó una senescencia lenta en el material de Alberite. Este episodio de contaminación tuvo su origen en la apertura de un nuevo canal de desagüe de aguas residuales, directamente al río, unos $50 \mathrm{~m}$ aguas arriba del lugar donde se encontraban situados los trasplantes. Dado que los briofitos son un grupo de plantas que carecen de espacios aéreos, la anoxia les afecta más acusadamente que a los macrófitos vasculares. Sin embargo. la especial arquitectura de los filidios de Fontiitalis antipyretica (aquillados y muy imbrica- dos) le confiere una cierta resistencia a la contaminación orgánica, aspecto previamente señalado por PEÑUELAS (1984).

Los valores de MDA obtenidos son sensiblemente inferiores a los señalados por DHINDSA \& MATOWE (1981) para otro briófito, Cratoneuron filicinum, sometido a sequía en laboratorio, pero las comparaciones son difíciles, ya que tanto el tipo de tensión como las especies y las condiciones ambientales son diferentes. Además, en el medio acuático la peroxidación de Iípidos quizá sea más difícilmente detectable que en el terrestre, ya que el deterioro de las membranas celulares puede ocasionar la pérdida de MDA por el lavado continuo a que está sometido el briófito en el río. Probablemente, en especies más sensibles a la contaminación, el MDA producido se liberaría más rápidamente (MARTINEZ ABAIGAR, 1989).

La elevada correlación de la peroxidación de lípidos con la DBO, en Alberite indica que la contaminación orgánica es la principal responsable de la senescencia de Fontinalis antipyretica. En Villanueva la peroxidación detectada estaría provocada por otros factores distintos de la contaminación, y la senescencia apuntada en la novena semana obedecería a otras causas inexplicadas.

Como conclusión, y al igual que ocurre en otros tipos de contaminación, la peroxidación de lípidos puede utilizarse como un indicador ecofisiológico de la senescencia provocada por la contaminación orgánica sobre briofitos acuáticos relativamente resistentes.

\section{AGRADECIMIENTOS}

Al Instituto de Estudios Riojanos, del cual fue becario JMA, y al Dr. Manuel Becana por su ayuda en la puesta a punto de la técnica.

\section{BIBLIOGRAFÍA}

ANDO, H. \& A. MATSUO, 1984. Applied Bryology. En: Advances in Bryology Vol. 2 (W. SCHULTZE-MOTEL, ed.) 133-229. J. Cramer. Vaduz.

BENSON-EVANS, K. \& P.F. WILLIAMS, 1976. Transplanting aquatic bryophytes to assess river pollution. $\mathbf{J}$. Bryol., 9: 81-92.

BROWN, D.H. 1984. Uptake of mineral elements and their use in pollution monitoring. En: The experimental biology of Bryophytes (A.F. DYER \& J.G. DUCKETT, eds.) 229-256. Academic Press. London.

BROWN, D.H., H. OUGHAM \& R.P. BECKETT, 1986. The effect of the herbicide dichlorophen on the physiology and growth of two bryophytes. Ann. Bot., 57: 201-209. 
CASE, J.W. \& H.R. KROUSE, 1980. Variations in sulfur content and stable sulfur isotope composition of vegetation near a sulfur dioxide source at Fox Creek, Alberta, Canada. Oecologia, 44: 248-257.

CHIA, L.S., C.I. MAYFIELD \& J.E. THOMPSON, 1984. Simulated acid rain induces lipid peroxidation and membrane damage in foliage. Plant Cell Environ., 7: 333-338.

DHINDSA, R.S. \& W. MATOWE, 1981. Drought tolerance in two mosses: correlated with enzymatic defence against lipid peroxidation.J. Exp. Bot., 32: 79-91.

DHINDSA, R.S., P. PLUMB-DHINDSA \& T.A. THORPE, 1981. Leaf senescence: correlated with increased levels of membrane permeability and lipid peroxidation, and decreased levels of superoxide dismutase and catalase. J. Exp. Bot., 32: 93-101.

DHINDSA, R.S., P. PLUMB-DHINDSA \& D.M. REID, 1982. Leaf senescence and lipid peroxidation. Effects of some phytohormones, and scavengers of free radicals and singlet oxygen. Physiol. Plant., 56: 453-457.

ELSTNER, E.F. 1982. Oxygen activation and oxygen toxicity. Ann. Rev. Plant Physiol, 33: 73-96.

EMPAIN, A. 1973. La vegetation bryophytique aquatique et subaquatique de la Sambre belge; son determinisme ecologique et ses relations avec des pollutions des eaux. Lejeunia, 69: $1-58$.

EMPAIN, A. 1978. Relations quantitatives entre les populations de bryophytes aquatiques et la pollution des eaux courantes. Definition d'un indice de qualite des eaux. Hydrobiologia, 60: 49-74.

GOOSENS, M. 1976 a. Croissance et vitalite de protonemas de bryophytes en atmosphere artificiellement polluee par le dioxide de soufre. Mem. Soc. R. Bot. Belg., 7: 87-100.

GOOSENS, M. 1976 b. Realisation de chambreties pour la culture de cryptogames en atmosphere artificiellement polluee. Bull. Soc. R. Bot. Belg., 109: 49-54.

GOOSENS, M. 1979. Comparaison de la sensibilite de neuf especes de bryophytes vis-a-vis du $\mathrm{SO}_{2}$. Bull. Soc. R. Bot. Belg., 112: 230-242.

HEATH, R.L. \& L. PACKER, 1965. Effect of light on lipid peroxidation in chloroplasts. Biochem. Biophys. Res. Comm., 19: 716-720.

HEATH, R.L. \& L. PACKER, 1968. Photoperoxidation in isolated chloroplasts I. Kinetics and stoichiometry of fatty acid peroxidation.Archs. Biochem. Biophys., 125: 189-198.

KIRCHMANN, R. \& J. LAMBINON, 1973. Bioindicateurs vegetaux de la contamination d'un cours d'eau par les effluents d'une centrale nucleaire a eau pressurisee. Bull. Soc. R. Bot. Relg., 106: 187-201.
LEBLANC, F. \& D.N. RAO, 1973. Effects of sulphur dioxide on lichen and moss transplants. Ecology, 54: 612-617.

LEBLANC, F. \& D.N. RAO, 1974. A review of the literature on Bryophytes with respect to air pollution. Bull. Soc. Bot. Fr., 121: 237-255.

LEBLANC, F. \& D.N. RAO, 1975. Effects of air pollutants on lichens and bryophytes. En: Responses of plants to air pollution (J.B. MUDD \& T.T. KOZLOWSKI, eds.) 237-272. Academic Press. London.

LEBLANC, F. \& J. DE SLOOVER, 1970. Relation between industrialisation and the distribution and growth of epiphytic lichens and mosses in Montreal. Can. J. Bot., 48: 1485-1496.

LESHEM, Y.Y. 1981. Oxy free radicals and plant senescence. What's New Plant Physiol., 12: 1-4.

MARGALEF, R. 1974. Ecología. Omega. Barcelona.

MARGALEF, R. 1983. Limnología. Omega. Barcelona.

MARTINEZ ABAIGAR, J. 1989. Briojitos acuáticos del río Iregua (La Rioja). Estudio florístico, ecológico y ecofisiológico. Respuestas a la contaminación orgánica. Tesis Doctoral. Universidad de Navarra.

MAUREL-KERMARREC, A., M. PALLY, L. FOULQUIER \& J.P. HEBRARD, 1983. Cinetique de la fixation d'un melange de Cesium 137, de Chrome 51, de Cobalt 60, de Manganese 54 et de Sodium 22 par Platyhypnidium riparioides (Hedw.) Dix. Cryptog. Bryol. Lichenol., 4: 299-313.

MÜHLE, H. 1984. Moose als Bioindikatoren. En: Advances in Bryology Vol. 2, (W. SCHULTZE-MOTEL, ed.) 65-89. J. Cramer. Vaduz.

PEÑUELAS, J. 1984. Pigments of aquatic mosses of the river Muga, NE Spain, and their response to water pollution. Lindbergia, 10: 127-132.

RAO, D.N. 1982. Responses of Bryophytes to Air Pollution. En: Bryophyte Ecology (A.J.E. SMITH, ed.) 445-472. Chapman \& Hall. London.

ROELOFS, J.G.M. 1986. The effect of airborne sulfur and nitrogen deposition on aquatic and terrestrial heathland vegetation. Experientia 42: 372-377.

SAWICKI, E., T.W. STANLEY \& H. JOHNSON, 1963. Comparison of spectrophotometric and spectrophotofluorometric methods for the determination of malondialdehyde. Analyt. Chem., 35: 199- 205.

SHIMAZAKI, K., T. SAKAKI, N. KONDO \& K. SUGAHARA, 1980. Active oxygen participation in chlorophyll destruction and lipid peroxidation in $\mathrm{SO}_{2}$-fumigated leaves of spinach. Plant Cell Physiol., 21: 1193-1204.

SUMMERLING, T.J. 1984. The use of mosses as indicators of airborne radionuclides near a major nuclear installation. Sci. Tot. Environ., 35: 251-266. 
WEHR, J.D., A. EMPAIN, C. MOUVET, P.J. SAY \& B.A. WHITTON, 1983. Methods for processing aquatic mosses used as monitors of heavy metals. Water Res., 17: 985-992.

WIELGOLASKI, F.E. 1975. Biological indicators of pollution. Urban Ecol., 1: 63-79.

WINNER, W.E. \& J.D. BEWLEY, 1978 a. Contrasts between bryophyte and vascular plants synecological responses in an $\mathrm{SO}_{2}$-stressed white spruce association in Central Alberta. Oecologia, 33: 311-325.
WINNER, W.E. \& J.D.BEWLEY, 1978 b. Terrestrial mosses as bioindicators of $\mathrm{SO}_{2}$ pollution stress. Oecologia, 35: 221-230.

WINNER, W.E. \& J.D. BEWLEY, 1983. Photosynthesis and respiration of feather mosses fumigated at different hydration levels with $\mathrm{SO}_{2}$. Can. J. Bot., 61: 1456-1461. 